\title{
Effect of sexual steroids on the calcium content of aortic atherosclerotic plaque of oophorectomized rabbits
}

\author{
J.M. Aldrighi ${ }^{1,2}$, \\ J.R. Lanz ${ }^{2}$, A.P. Mansur², \\ T.L.R. Martinez ${ }^{2}$ \\ and J.A.F. Ramires ${ }^{2}$
}

${ }^{1}$ Faculdade de Saúde Pública and ${ }^{2}$ Instituto do Coração, Faculdade de Medicina, Universidade de São Paulo, São Paulo, SP, Brasil

\section{Correspondence \\ J.M. Aldrighi \\ Rua Afonso de Oliveira Santos, 50 \\ 05663-030 São Paulo, SP \\ Brasil \\ Fax: +55-11-3744-7890 \\ E-mail: jmaldri@usp.br}

Publication supported by FAPESP.

$\ldots \ldots \ldots \ldots \ldots \ldots \ldots \ldots$

Received March 22, 2004

Accepted October 13, 2004

\begin{abstract}
We determined the effect of conjugated equine estrogen plus medroxyprogesterone acetate on calcium content of aortic atherosclerotic lesions in oophorectomized adult New Zealand rabbits submitted to a cholesterol rich diet. Five groups of 10 animals each were studied: $\mathrm{G} 1$ = control, $\mathrm{G} 2$ = cholesterol diet only, G3 = diet plus conjugated equine estrogen $(0.625 \mathrm{mg} / \mathrm{day}) ; \mathrm{G} 4$ and G5 = diet, conjugated equine estrogen $(0.625 \mathrm{mg} /$ day) plus medroxyprogesterone acetate (5 and 10 $\mathrm{mg} /$ day, respectively). Mean weight varied from $2.7 \pm 0.27$ to $3.1 \pm$ $0.20 \mathrm{~kg}(\mathrm{P}=0.38)$ between groups at the beginning and $3.1 \pm 0.27$ to $3.5 \pm 0.20 \mathrm{~kg}(\mathrm{P}=0.35)$ at the end of the experiment. Cholesterol and triglyceride levels were determined at the time of oophorectomy, 21 days after surgery (time 0 ), and at the end of follow-up of 90 days. The planimetric method was used to measure plaque and caryometric method for histopathologic examination of the aorta. Calcium content was determined by the method of von Kossa. A similar increase in cholesterol occurred in all treated groups without differences between them at the end of the study. Groups G4 and G5 had smaller areas of atherosclerotic lesions $\left(2.33 \pm 2.8\right.$ and $2.45 \pm 2.1 \mathrm{~cm}^{2}$, respectively) than the groups receiving no progestogens (G2: $5.6 \pm 4$ and G3: $4.6 \pm$ $2.8 \mathrm{~cm}^{2} ; \mathrm{P}=0.02$ ). The relation between lesion area and total aorta area was smaller in groups treated with combined drugs compared to the groups receiving no progesterone (G4: $14.9 \pm 13$ and G5: 14.2 \pm 13.4 vs $\mathrm{G} 2: 35.8 \pm 26$ and $\mathrm{G} 3: 25 \pm 8 \mathrm{~cm}^{2}$, respectively; $\left.\mathrm{P}=0.017\right)$. Oral conjugated equine estrogen $(0.625 \mathrm{mg} /$ day $)$ plus medroxyprogesterone acetate $(5$ or $10 \mathrm{mg} /$ day $)$ provoked a greater reduction in atherosclerotic plaque area and calcium content in treated groups, suggesting a dose-dependent effect.
\end{abstract}

Key words

- Estrogens

- Progestagens

- Calcium

- Atherosclerosis

- Oophorectomy

- Aortic plaque area

\section{Introduction}

Menopause, the end of menses in a woman's life, is characterized by the exhaustion of ovarian follicles, resulting in a reduction of sexual steroids (mainly estrogen) and in the occurrence of uncomfortable symptoms and serious diseases, such as osteoporosis and cardiovascular disease (CVD). CVD not only is the major cause of death in industrialized societies but also is an important women's public health problem worldwide, 
including Brazil (1-3).

In experimental studies on animals and in clinical studies on humans, estrogen has been implicated as a protector against CVD; in fact, the risk of CVD is seven times higher in oophorectomized women than in intact women (4). Randomized clinical studies and a recent analysis (5-9) did not show benefits of hormone replacement therapy after menopause regarding primary and secondary prevention of CVD. This outcome is different from that of observational epidemiologic and animal studies in which estrogen was found to promote inhibition of atherosclerotic plaque $(10,11)$.

In humans, few clinical studies have shown secondary prevention benefits. The results of the Heart and Estrogen/Progestin Replacement Study (HERS) (5) and the Effects of Estrogen Replacement on the Progression of Coronary-Artery Atherosclerosis Study (ERA) (7) revealed that the combination of conjugated equine estrogen (CEE) with medroxyprogesterone acetate (MPA) does not reduce the risk of CVD. However, it is important to note that the average age of women included in these studies was more than 62 years, with the subjects already exhibiting a severe degree of atherosclerosis.

In recent research on humans (12), progestrogens did not have a beneficial effect on any study participant when combined to estrogens. Benefit was seen only in the initial stages of the atherogenic process (i.e, in women between 40 and 60 years of age); furthermore, in advanced CVD when complications can occur (i.e, women $\geq 65$ years) estrogen seems to have harmful effects on cardiovascular events.

However, since postmenopausal women with an intact uterus must be treated with a combination of progestagen and estrogen to reduce the risk of hyperplasia and endometrial cancer, one question emerges: might the addition of different progestagens to hormonal replacement therapy with estrogen have harmful effects on the cardiovascular sys- tem? Such effects were shown in the arm of the Women's Health Iniciative Study (WHI) (6) in which women were prescribed CEE plus MPA and presented a high risk of CVD and breast cancer; as a result, this arm of the WHI study was stopped.

In the present investigation, we used the same hormonal combination as used in the HERS (5) and WHI (6) studies in order to evaluate the calcium content of atherosclerotic plaques in rabbits.

\section{Material and Methods}

Fifty 6-month-old adult female New Zealand white rabbits were used in the study. Mean weight varied from $2.7 \pm 0.27$ to $3.1 \pm$ $0.20 \mathrm{~kg}(\mathrm{P}=0.38)$ between groups at the beginning and $3.1 \pm 0.27$ to $3.5 \pm 0.20 \mathrm{~kg}$ (P $=0.35)$ at the end of the experiment. After 7 days of adaptation to the laboratory, all animals underwent oophorectomy to simulate menopause. On the 21 st postoperative day (time 0 ), all animals were randomly divided into 5 groups: G1 (control), animals fed a normal diet (normal ration); G2, animals fed a cholesterol-rich diet (0.5\%); G3, animals fed a cholesterol-rich diet $(0.5 \%)$ in combination with CEE (0.625 mg/day); G4, animals fed a cholesterol-rich diet $(0.5 \%)$ and a combination of CEE $(0.625 \mathrm{mg} /$ day $)$ plus MPA ( $5 \mathrm{mg} /$ day); G5, same treatment as G4 $(0.625 \mathrm{mg} /$ day CEE) plus MPA $(10 \mathrm{mg} /$ day $)$. Hormone administration was accomplished by adding $0.625 \mathrm{mg} /$ day CEE to $20 \mathrm{~g}$ of ration before diluting the hormone in a mix of alcohol and water $50 \%$ each. The same method was used for addition of MPA ( 5 and $10 \mathrm{mg} /$ day) to $50 \mathrm{~g}$ of ration.

All work was conducted according to accepted regulations of animal experimentation.

The total duration of treatment was 90 days. Serum lipids (total cholesterol and triglycerides) were measured at the time of surgery (time -21), 21 days after surgery (randomization, time 0 ), and after 90 days of 
follow-up (time 90). The aortas were dissected, fixed, and stained with Sudan III for microscopic visualization of the lesions, and the percentage of the total area of the aorta occupied by lesions was quantified by computerized planimetric measurements.

The results are reported as percent area of compromised aorta in relation to total aorta area. Three segments of a transverse cut of the thoracic aorta (the first $2 \mathrm{~cm}$ ) and of the abdominal aorta (first 7 to $12 \mathrm{~cm}$ ) were embedded in paraffin, cut into 5 - $\mu \mathrm{m}$ sections and stained with hematoxylin and eosin. The sections were then examined morphometrically to determine the height of the lesions (either the intimal value or the average) and for histopathological analysis of the intimal and middle layers. To evaluate the presence of calcium, the segments were stained by the method of von Kossa. The relation between the inner and middle layers was also determined.

Group data were compared by the Kruskal-Wallis test. The parameters were measured twice in the same animal and compared using the Wilcoxon signed rank test. Data for the variables for which more than two measures were obtained (at the time of surgery, 21 days after surgery, and after 90 days of follow-up) were analyzed by the non-parametric Friedman test. The level of significance was set at 0.05 for all analyses.

\section{Results}

From a total of 50 animals, 47 completed the experiment, two rabbits of G4 and one of G5 died during the study, one due to traumatic injury in the cage and two due to infection of the abdominal wound. The basal weight at the beginning of the experiment and the weight gain throughout the study did not differ between groups.

\section{Cholesterol levels}

The cholesterol levels of all treated groups differed from control (G1) throughout the 90-day treatment $(\mathrm{P}<0.001$, Table 1$)$. G3 and G4 exhibited the lowest values at the beginning of the experiment before randomization, and were also the groups with the highest increase at the end of the experiment (Table 1). Furthermore, G1 remained unaffected at time 0 and 90 days; however, all treated groups showed a significant rise in mean cholesterol levels at time 0 and 90 days and this increase was up to 10-13 times the initial levels in G4 and G5 ( $\mathrm{P}<0.005$, Table 1).

\section{Triglyceride levels}

There was a great variability in triglyceride levels amongst groups at the beginning of the study with a large standard deviation. These values increased when comparing time -21 and time 90 , with G4 having the greatest increase (Table 2).

\section{Evaluation of aortic lesions}

Intense lipid deposition was observed macroscopically in the aortic arch and abdominal and thoracic aortas of G2, G3, G4, and G5, which differed from G1, in which atherosclerotic plaques were not shown. There were no evident macroscopic hypercholesterolemic differences among groups, but marked individual variability was observed. The total area of compromised aor-

Table 1. Serum cholesterol levels at surgery (21 days before randomization: -21), 21 days after surgery (time of randomization: 0), and at the end of follow-up (90).

\begin{tabular}{|c|c|c|c|}
\hline \multirow[t]{2}{*}{ Group } & \multicolumn{3}{|c|}{ Time (days) } \\
\hline & -21 & 0 & 90 \\
\hline 1 & $131.2 \pm 43$ & $112.3 \pm 35$ & $185.0 \pm 73$ \\
\hline 2 & $105.9 \pm 67$ & $96.9 \pm 30$ & $984.8 \pm 293$ \\
\hline 3 & $78.6 \pm 28$ & $86.3 \pm 28$ & $1100.7 \pm 401$ \\
\hline 4 & $58.6 \pm 23$ & $101.5 \pm 47$ & $1420.5 \pm 496$ \\
\hline 5 & $118.7 \pm 47$ & $84.7 \pm 25$ & $976.8 \pm 160$ \\
\hline$P$ value & $<0.05$ & $<0.37$ & $<0.01$ \\
\hline
\end{tabular}

Data are reported as means $\pm \mathrm{SD}(\mathrm{mg} / \mathrm{dl})$. Kruskal-Wallis test $\mathrm{H}$ in each time course was time $-21=15$, time $0=4$ and time $90=26$. 
tas ranked from 11.88 to $18.1 \mathrm{~cm}^{2}$ with a mean of $14.9 \pm 2 \mathrm{~cm}^{2}$ in $\mathrm{G} 1$, which presented the minimum values to the group with the highest values (G4) which ranked from 14.23 to $23.7 \mathrm{~cm}^{2}$ and a mean of $17.8 \pm 3.3 \mathrm{~cm}^{2}(\mathrm{P}$ $>0.05$ ).

\section{Planimetric study}

Excluding the control group, no difference in total aorta area was observed among groups. Analysis of atherosclerotic plaques revealed that both groups treated with progestagens (G4 and G5) had smaller areas of atherosclerotic lesions $(2.33 \pm 2.8$ and $2.45 \pm$ $2.1 \mathrm{~cm}^{2}$, respectively) than the groups receiving no progestagens (G2: $5.6 \pm 4$ and $\left.\mathrm{G} 3: 4.6 \pm 2.8 \mathrm{~cm}^{2} ; \mathrm{P}=0.02\right)$. In other words,

$\begin{aligned} & \text { Table 2. Serum triglyceride levels at surgery (21 days before randomization: }-21), 21 \\
& \text { days after surgery (time of randomization: } 0), \text { and the end of follow-up (90). }\end{aligned}$
\begin{tabular}{lccc} 
Group & \multicolumn{4}{c}{ Time (days) } \\
\cline { 2 - 4 } & -21 & 0 & 90 \\
\hline 1 & $127.0 \pm 57$ & $107.5 \pm 33$ & $148.0 \pm 10$ \\
2 & $71.9 \pm 34$ & $110.6 \pm 30$ & $130.2 \pm 65$ \\
3 & $71.5 \pm 17$ & $88.6 \pm 20$ & $145.5 \pm 118$ \\
4 & $81.5 \pm 16$ & $81.5 \pm 21$ & $192.9 \pm 76$ \\
5 & $147.0 \pm 40$ & $125.6 \pm 67$ & $172.4 \pm 111$ \\
P value & $<0.001$ & $<0.03$ & $>0.05$
\end{tabular}

Data are reported as means $\pm \mathrm{SD}(\mathrm{mg} / \mathrm{dl})$. Kruskal-Wallis test $\mathrm{H}$ in each time course was time $-21=15$, time $0=4$ and time $90=26$.

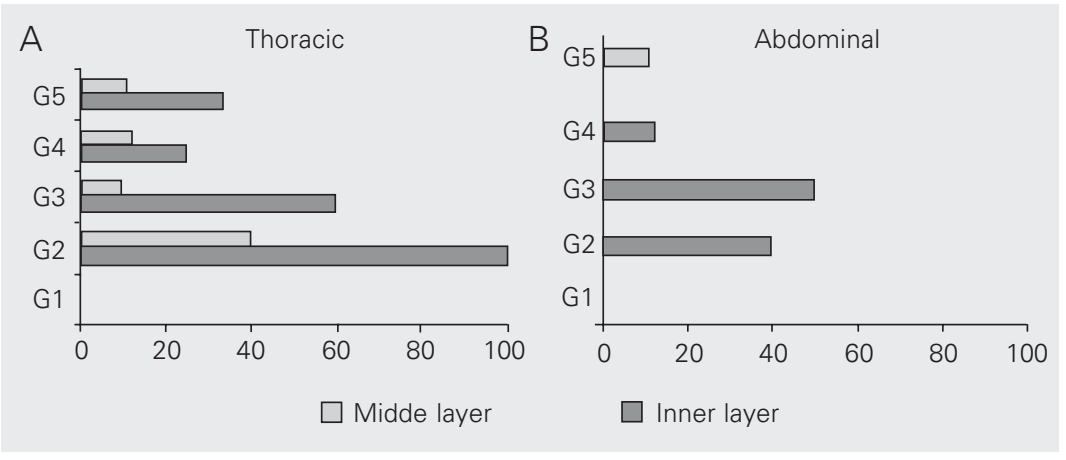

Figure 1. Calcium content of the inner and middle layers of thoracic $(A)$ and abdominal aortic (B) segments. Data are reported as percent of calcium content in each layer. Thoracic segment: $\chi^{2}=23.13, P=0.0001(G 1=G 4=G 5) \neq G 2 \neq G 3$. Abdominal segment: inner layer $\chi^{2}=12.52, P=0.0139 *(G 1=G 4 V) \neq(G 2=G 3)$; middle layer not significant. a 43 and $45 \%$ reduction of atherosclerotic plaque was observed in the groups submitted to combined treatment. The relationship between lesion area and total aorta area was smaller for the groups treated with combined drugs compared to the groups receiving no progesterone (G4: $14.9 \pm 13$ and G5: $14.2 \pm$ 13.4 vs G2: $35.8 \pm 26$ and G3: $25 \pm 8 \mathrm{~cm}^{2}$, respectively; $\mathrm{P}=0.017$ ).

\section{Calcium content}

Calcium deposits were detected by Von Kossa's stain in the inner layer of the thoracic aortas in $100 \%$ of animals fed the cholesterol-rich diet compared with $40 \%$ in the middle layer. The groups treated with estrogens showed a $60 \%$ reduction of calcium content in the inner layer and in those groups treated with estrogen plus progestogen there was an even greater reduction in calcium content when compared to G2,75\% in $\mathrm{G} 4$, and $67 \%$ in G5 $(\mathrm{P}<0.05)$. The calcium content of the abdominal aorta was greater in G2 and G3 (40 and 50\%, respectively). In G4 and G5 the reduction in calcium content was 12.5 and $0 \%$, respectively. In the middle layer of the abdominal aorta, the expression of calcium was even smaller, with a low percentage in G5 and no expression in the other groups (Figure 1).

\section{Discussion}

The cholesterol-rich diet was sufficient to provoke atherosclerotic lesions in oophorectomized animals after 3 months, a fact already demonstrated in other protocols (13). Despite the clear relation between the increase in cholesterol and the presence of atherosclerotic plaque, it is important to note that cholesterol levels of the groups treated with CEE (G3) or estrogen associated with progestagens (G4 and G5) were not different at the end of the study. However, the total area of aortic lesion was smaller in G3 than that in G4 and G5; in other words, the results 
obtained for the groups treated with estrogen plus progestagens suggested an effect of the hormones on plaque characteristics independently of the circulating levels of cholesterol.

The HERS (5) detected a $29 \%$ increase in cardiovascular events among users of combined drugs (CEE plus MPA) administered over a long period of time for secondary prevention of CVD in post-menopausal women with an intact uterus, when compared with placebo. The HERS deserves some criticism. First, the women studied were older (average, 63.3 years), a period of time when tromboembolic events are more frequent; second, the control group used statins to a greater extent, and third the doses of steroids prescribed were high. In fact, regarding the steroid doses, it is known that estrogens, depending on the type, dose and method of administration increase high density lipoprotein (14) and reduce low density lipoprotein (15), while progestagens, depending on the type and dosage, can antagonize the beneficial effects of estrogen.

Our results showing plaque characteristics using a combination of CEE and MPA were similar when compared with their effects on the height of lesions in the middle layer of the aortic thoracic segment in the group treated with the smaller dose of progestagen (G4). It is known that the formation of atherosclerotic plaque is directly linked to cholesterol levels. In the present study, this relation was also expected but, in fact, it did not occur. Possible explanations for this outcome include the antioxidant effects of estrogens and other effects such as alterations in the catabolism of low density lipoprotein (14), increase in high density lipoprotein (15), disturbance in the production of nitric oxide $(16,17)$ and the antiatherogenic effects of estrogens, like those related to cellular proliferation of the neointima $(18,19)$.

A recent experiment (20) showed that the combination of CEE and micronized proges- terone in a model of hormonal replacement treatment reduced the levels of monocyte chemoattractant factor (MCP-1), which is one of the decisive factors in the formation of plaque. In our study, the addition of a fixed dose of progestagen did not alter the benefit obtained with estrogen alone, suggesting the possibility of the existence of an ideal dose of progestagen that does not limit the protective action of estrogen.

When the lesions induced in the aorta were evaluated by the computerized planimetric technique, it was observed that the lesion area of animals treated with estrogen alone did not decrease, a result differing from those obtained by others in similar experiments (21-23). However, the animals treated with progestins at the doses of 5 and $10 \mathrm{mg}$ in combination with estrogens (G4 and G5) showed a significant decrease in atherosclerotic areas. We do not have an explanation for this observation. Additional studies should be conducted to analyze other systems such as the renin-angiotensin system, whose proliferative actions are mediated by AT1 receptors (modulated by estrogen) $(24,25)$.

The natural evolution of atherosclerotic plaque involves an increase in calcium, which is the major component of plaque and which is more indicative of old organized plaque. In our study, the evolution of atherosclerotic plaque was reproduced within a short period of time, and the groups treated with MPA showed a surprising reduction in calcium content in the atherosclerotic plaque.

Our data agree with those of clinical studies (26) that evaluated calcium content in the coronary arteries of women and men and observed a significant reduction in women up to 60 years of age, suggesting a possible relation with hormonal steroids.

Another study (27) evaluating by standard computed tomography the calcium content of the coronary arteries of postmenopausal women matched with a control group with the same risk factors for atherosclero- 
sis, verified that hormone therapy users (independent of the type of hormone used) had a smaller calcium content (14\%) than the control group (43.2\%) during the first year of treatment.

The inhibition of the atherosclerosis process observed in the present study in rabbits with a combination of estrogens plus progestagens at the dose of $5 \mathrm{mg}$ /day agrees with the results obtained by Spagnoli et al. (13), who reported inhibition of the atherosclerotic process in the aorta of rabbits with the use of two progestagens (norethisterone and MPA), independently of the deleterious influences on lipid levels. However, it should be emphasized that none of the studies conducted to date have reported a reduction in the calcium content of atherosclerotic plaques with the use of MPA.

The present results allow us to conclude that treatment with estrogens alone in the formulation used in this experiment did not modify the percent of the lesion area in the aorta, although it promoted an increase in the height of the inner layer of the abdominal segment. In contrast, the CEE + MPA combination ( 5 and $10 \mathrm{mg} /$ day) reduced the lesion area in all aortic segments studied.

The combination of estrogen with a lower progestagen dosage $(5 \mathrm{mg}$ /day) promoted a significant decrease in the height of the lesions in the medial layer of the thoracic segment. The calcium content of the thoracic aorta was significantly reduced when progestagen was added to the treatment, in contrast to the increase seen in the abdominal aorta.

The results of our study suggest that the effect of progestagen in the form of MPA may be dose dependent. Small doses in combination with a fixed dose of estrogens $(0.625$ $\mathrm{mg} /$ day) had the most beneficial effect on the aorta. New studies should be conducted with other estrogen combinations and progestagens using different doses and routes of administration to define the limit between protection and cardiovascular risk in the patients for whom hormonal replacement therapy should be indicated.

\section{References}

1. Mansur A, Favarato D, de Souza MF, Avakian SD, Aldrighi JM, César LA \& Ramires JA (2001). Trends in death from circulatory diseases in Brazil between 1979 and 1996. Arquivos Brasileiros de Cardiologia, 76: 497-510.

2. Lotufo PA (1998). Premature mortality from heart diseases in Brazil. A comparison with other countries. Arquivos Brasileiros de Cardiologia, 70: 321-325.

3. Lessa I (2002). Trends in productive years of life lost to premature mortality due to coronary heart disease. Arquivos Brasileiros de Cardiologia, 79: 617-622.

4. Stampfer MJ, Colditz GA \& Willett WC (1990). Menopause and heart disease: a review. Annals of the New York Academy of Sciences, 592: 193-203.

5. Herrington DM (1999). The HERS trial results: paradigms lost? Heart and Estrogen/Progestin Replacement Study. Annals of Internal Medicine, 131: 463-466.

6. Rossouw JE, Anderson GL, Prentic RL et al. (2002). Risks and benefits of estrogen plus progestin in healthy postmenopausal women: Principal results from the women's health initiative randomized controlled trial. Journal of the American Medical Association, 288: 321-333.

7. Herrington DM, Reboussin DM, Brosnihan KB et al. (2000). Effects of estrogen replacement on the progression of coronary-artery atherosclerosis. ERA trial. New England Journal of Medicine, 343: 522529.

8. Abramson BL (2002). Postmenopausal hormone replacement therapy and the prevention of cardiovascular disease: a review. Journal of Cardiovascular Risk, 9: 309-314.

9. Adams MR, Williams JK, Clarkson TB \& Jayo MJ (1991). Effects of oestrogens and progestagens on coronary atherosclerosis and osteoporosis of monkeys. Baillieres Clinical Obstetrics and Gynaecology, 5: 915-934.

10. Adams MR, Anthony MS, Manning JM, Golden DL \& Parks JS (2000). Low-dose contraceptive estrogen-progestin and coronary artery atherosclerosis of monkeys. Obstetrics and Gynecology, 96: 250-255.

11. Clarkson TB \& Appt SE (2003). MPA and postmenopausal coronary artery atherosclerosis revisited. Steroids, 68: 941-951.

12. Mikkola TS \& Clarkson TB (2002). Estrogen replacement therapy, atherosclerosis, and vascular function. Cardiovascular Research, 53: 605-619.

13. Spagnoli LG, Palmieri G, Mauriello A, Orlandi A, Bancheri C \& Pasetto N (1990). High-dose synthetic progestagens inhibit foam and smooth muscle cell proliferation and atherosclerotic plaque 
formation in aortas of rabbits fed a hypercholesterolemic diet. Atherosclerosis, 82: 27-36.

14. Campos H, Walsh BW, Judge H \& Sacks FM (1997). Effect of estrogen on very low density lipoprotein and low density lipoprotein subclass metabolism in postmenopausal women. Endocrinology and Metabolism, 82: 3955-3963.

15. Nanda S, Gupta N, Harish C, Sangwani M \& Sangwani K (2003). Effect of oestrogen replacement therapy on serum lipid profile. Australian and New Zealand Journal of Obstetrics and Gynaecology, 43: 213-216.

16. Gong M, Wilson M, Kelly T et al. (2003). HDL-associated estradiol stimulates endothelial NO synthase and vasodilation in an SR-BIdependent manner. Journal of Clinical Investigation, 111: 15791587.

17. Takeda-Matsubara $Y$, Nakagami H, Iwai M, Cui T-X, Shiuchi T, Akishita M, Nahmias C, Ito M \& Horiuchi M (2002). Estrogen activates phosphatases and antagonizes growth-promoting effect of angiotensin II. Hypertension, 39: 41-45.

18. Collins P (2001). Vascular effects of hormones. Maturitas, 38: 4550.

19. Liu H-W, Iwai M, Takeda-Matsubara Y, Wu L, Liz J-M, Okumura M, Cui T-X \& Horiuchi M (2002). Effect of estrogen and AT1 receptor blocker on neointima formation. Hypertension, 40: 451-457.

20. Koh KK, Son JW, Ahn JY, Lee SK, Hwang HY, Kim DS, Jin DK, Ahn TH \& Shin EK (2001). Effect of hormone replacement therapy on nitric oxide bioactivity and monocyte chemoattractant protein-1 levels. International Journal of Cardiology, 81: 43-50.
21. Kushwara RS \& Hazzard W (1981). Exogenous estrogens attenuate dietary hypercholesterolemia in the rabbit. Metabolism, 30: 359366.

22. Harabo J, Hansen BF \& Christiansen C (1991). Hormone replacement therapy prevents coronary artery disease in ovariectomized cholesterol fed rabbits. Acta Pathologica, Microbiologica et Immunologica Scandinavica, 99: 721-727.

23. Henrikson $P$, Stamber $M$, Eriksson M, Rudling M, Diczfaluzy U, Berglund L \& Angelin B (1989). Oestrogen-induced changes in lipoprotein metabolism: role in prevention of atherosclerosis in the cholesterol-fed rabbit. European Journal of Clinical Investigation, 19: 395-403.

24. Hough JL \& Zilversmith BD (1986). Effect of 17-beta estradiol on aortic cholesterol content and metabolism in cholesterol-fed rabbits. Atherosclerosis, 6: 57-63.

25. Nickening G, Strehlow K, Wassmann S, Bäumer A, Albory K, Sauer $H$ \& Böhm M (2000). Differential effects of estrogen and progesterone on AT1 receptor gene expression in vascular smooth muscle cells. Circulation, 102: 1828-1833.

26. Janowitz WR, Agaston AS, Kaplan G \& Viamonte Jr M (1993). Difference in prevalence and extent of coronary artery calcium detected by ultrafast computed tomography in asymptomatic men and women. American Journal of Cardiology, 72: 247-254.

27. Shemesh J, Frenkel Y, Leibovitch L, Grossman E, Pines A \& Motro $M$ (1997). Does hormone replacement therapy inhibit coronary artery calcification? Obstetrics and Gynecology, 89: 989-992. 